
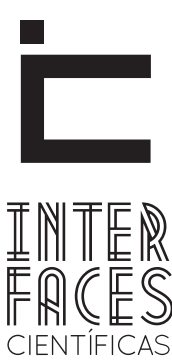

SAÚDE E AMBIENTE

\title{
AVALIAÇ̃̃o DA POLUIÇÃO ATMOSFÉRICA ORIUNDA DE INDÚSTRIA CIMENTEIRA E IMPACTO NA SAÚDE
}

\author{
Angélica B. Ferreira ${ }^{1}$ \\ Sidnei O. Souza ${ }^{3}$ \\ José do Patrocínio H. Alves
}

\author{
José 0. Santos ${ }^{2}$ \\ Wilson N. S. Júnior
}

\section{RESUMO}

A população dos povoados Machado, Estivas e Estivas II, localizados em Laranjeiras, e Nossa Senhora do Socorro, Sergipe, região na qual está inserido um grande complexo industrial cimenteiro, sofre com a poluição do ar produzida por estas indústrias. 0 objetivo deste trabalho é avaliar o risco da exposição humana às emissões atmosféricas poluentes deste complexo industrial cimenteiro, por meio de biomonitoramento passivo, utilizando cascas de árvores presentes na região de estudo como bioindicadores, como alternativa de monitoramento abrangente, preciso e de baixo custo de poluentes do ar. Para esta proposta, concentrações de elementos químicos foram determinadas pela técnica do EDXRF, nas cascas de árvores. Foram calculados os fatores de enriquecimento para os elementos encontrados nas cascas da região em estudo, com o objetivo de verificar aqueles que são de inserção de atividades antropogênicas. Mapas de atenuação das concentrações dos elementos foram feitos com o intuito de identificar "hor spots" e de conhecer a mobilidade do elemento no ar. Foram coletados dados de saúde da população nos postos de saúde dos povoados Machado, Estivas e Estivas II . Dados de saúde dos pacientes do povoado Oiteiros, localizado em Nossa Senhora do Socorro e livre das emissões aéreas das indústrias de cimento foram coletados no posto de saúde local para comparação com os dados de saúde dos povoados impactados pelas cimenteiras. Os resultados mostram ser necessário, que sejam efetuados estudos epidemiológicos, importantes para proteção da saúde e estabelecimento de medidas mitigadoras para esta população exposta às concentrações mais elevadas de poluentes.

\section{PALAVRAS-CHAVE}

Cascas de Árvores. EDXRF. Dados de Saúde. 


\section{ABSTRACT}

The population of Machado municipalities, Estivas and Estivas II, located in Laranjeiras city, and Nossa Senhora do Socorro city (Sergipe State, northeast Brazil), region in which a large cement industrial complex, suffers from air pollution produced by these industries. The objective of this study is to assess the risk of human exposure to the pollutant atmospheric emissions from the cement industrial complex, through passive biomonitoring using tree bark, in the region of study, as bioindicators, because it is a comprehensive, accurate and low cost alternative to monitor air pollutants. For this proposal, concentrations of chemical elements were determined by EDXRF technique, in the tree barks. The enrichment factors were calculated for the elements found in the barks from the study area attempting to verify which came from insertions on anthropogenic activities. Attenuation maps of the concentrations of the elements were made in order to identify "hot spots" meet and mobility of the element in ar. Attenuation maps of the concentrations of the elements were made in order to identify "hor spots" and meet the mobility of the element in the air. Population health data at posts in Machado, Estivas and Estivas II villages were collected. Population health data collected from clinics in the Machado, Estivas and Estivas II municipalities. Patientes health data from Oiteiros municipality located in Nossa Senhora do Socorro city and free of air emissions from cement industries were collected at the free health centre for comparison with the data of the villages affected by cement industries. The results show that it is necessary epidemiological studies, for health protection and establishment of mitigation measures for this population exposed to the highest concentrations of pollutants.

\section{KEYWORDS}

Tree Barks. EDXR. Health Data.

\section{RESUMEN}

La población de las aldeas Machado, Estivas y Estivas II, ubicadas en Laranjeiras, y Nossa Senhora do Socorro, región de Sergipe en el que un sitio grande planta cementera se encuentra, sufre de la contaminación del aire producida por estas industrias. El objetivo de este estudio es evaluar el riesgo de exposición humana a este cemento contaminante emisiones atmosféricas complejos industriales a través de la vigilancia biológica pasiva utilizando corteza en la región de estudio como bioindicadores, como alternativa a, un seguimiento preciso integral y bajo costo Los contaminantes del aire. Para esta propuesta, las concentraciones de los elementos químicos se determinaron por la técnica EDXRF, en la corteza de los árboles. Se calcularon los factores de enriquecimiento de los elementos que se encuentran en la corteza de la zona de estudio, con el objetivo de verificar la inserción de las actividades antropogénicas. Mapas de atenuación de las concentraciones de los elementos se hicieron con el fin de identificar los "puntos hor " se reúnen y la movilidad del elemento en los datos recogidos ar. Fueron colectados en clínicas de salud de la población en los pueblos Machado, Estivas y Estivas II, Los datos de salud de los pacientes Oiteiros la aldea, ubicada en Nossa Senhora do Socorro y libre de emisiones de contaminantes a la atmósfera, procedentes de las in- 
dustrias de cemento y se recogieron en el puesto de salud local para la comparación con los datos de las aldeas afectadas por la salud de cemento. Los resultados muestran que es necesario un estudio epidemiológico importante, para la protección de la salud y el establecimiento de medidas de mitigación para esta población expuesta a las concentraciones más altas de contaminantes estudios se llevan a cabo.

\section{PALABRAS CLAVE}

Cortezas de Árboles. EDXRF. Datos de Salud.

\section{INTRODUÇ̧̃̃O}

A população dos povoados Machado, Estivas e Estivas II, localizados, o primeiro no município de Laranjeiras, Sergipe e o segundo e terceiro em Nossa Senhora do Socorro, cidade vizinha de Laranjeiras, região na qual está inserido um grande complexo industrial cimenteiro, formado por dois dos maiores conglomerados da região nordeste do Brasil, que chegam a produzir 240 mil toneladas de cimento ao mês, sofre com a poluição do ar produzida por este complexo cimenteiro. Apesar disto, a área de influência das emissões destas duas indústrias e seus possíveis efeitos sobre a saúde da população não têm sido verificadas, uma vez que medições da poluição do ar não estão disponíveis nesta área. Na verdade, esta é uma situação bastante comum nos países em desenvolvimento, onde importantes fontes de poluição do ar podem ocorrer em áreas que não possuem o devido monitoramento destes contaminantes.

Nesse contexto, a simplificação dos métodos de medição de partículas no ambiente, tal como a acumulação de elementos traço em cascas de árvores pode fornecer informações relevantes na determinação da área de influência da fonte emissora de poluição e a identificação destes hot spots de poluição, importante para a proteção da saúde e estabelecimento de medidas mitigadoras para este segmento da população exposto às concentrações mais elevadas destes poluentes.

\section{MÉTODO}

\section{1 ÁREA DE ESTUDO}

O povoado Machado está inserido na área rural do município de Laranjeiras, Sergipe, que está localizada a uma latitude $10^{\circ} 48^{\prime} 23^{\prime \prime}$ sul e a uma longitude $37^{0} 10^{\prime} 12$ " oeste, à 23 km da capital, Aracaju. A cidade ocupa uma área de $163,4 \mathrm{~km}^{2}$ e possui uma população de 23.923 habitantes, com uma densidade populacio- nal de 165,52 habitantes/ $/ \mathrm{km}^{2}$ (IBGE, 2011). Os povoados Estivas e Estivas II estão inseridos na área rural do município de Nossa Senhora do Socorro, cidade vizinha a Laranjeiras. Nossa Senhora do Socorro está localizada a uma latitude $10^{\circ} 51^{\prime} 18^{\prime}$ ' sul e a uma longitude $37^{\circ} 07^{\prime} 34^{\prime \prime}$ oeste, à 8 km de Aracaju. Ocupa uma 
área de 157,2 km² e possui uma população de 155.334 habitantes, com uma densidade populacional de 1. 021, 04 habitantes/km² (IBGE, 2011).

\subsection{AMOSTRAGEM DAS CASCAS DE ÁRVORES}

Foram coletadas cascas de 64 árvores na região que compreende os povoados Machado, Estivas, Estivas II e também as duas fábricas de cimento, de setembro a novembro de 2010. Para cada árvore amostrada, foram gravadas coordenadas usando o Sistema de Posição Global para coordenadas Universal Transverse Mercator (UTM). A coleta das cascas levou em consideração a direção preferencial dos ventos (SE durante 8 meses e L durante 4 meses do ano (SILVA et al, 2002) que passa na região das fábricas de cimento.

\subsection{AMOSTRAGEM DO CIMENTO}

Foram analisadas, também, amostras de cimento produzido pelas fábricas para determinação dos elementos constituintes das amostras.

\subsection{PROCEDIMENTO DE COLETA E TRATAMENTO DAS AMOSTRAS DE CASCAS DE ÁRVORES PARA ANÁLISE}

As cascas removidas dos troncos das árvores, usando uma faca afiada foram guardadas em sacos de papel. No tratamento destas amostras foram retirados eventuais liquens ou/e musgos nelas presentes. As amostras não foram lavadas para não haver perda do material de interesse (que foi adsorvido nas cascas). Para análise, a parte superficial externa das cascas (cerca de $3 \mathrm{~mm}$ ) (SCHELLE et al., 2001) foi removida com o uso de um ralador de titânio (99\% de pureza para não haver contaminação) e triturado em almofariz de ágata. O material obtido foi passado em peneira de 170 mesh de modo a obter a casca na forma de pó. Uma massa de 0,5 a $0,6 \mathrm{~g}$ da amostra e de $3,0 \mathrm{~g}$ de ácido bórico $\left(\mathrm{H}_{3} \mathrm{BO}_{3}\right.$ p.a.) foram colocadas em um cilindro, e prensadas por 40 segundos com a ajuda de uma prensa de 20 toneladas de força, para obter pastilhas de dupla camada (amostra de casca e ácido bórico) com 30 $\mathrm{mm}$ de diâmetro. As pastilhas preparadas foram guardadas em dessecador, contendo sílica e posteriormente foram analisadas pelo método de espectrometria de fluorescência de raios $X$ por dispersão de energia (EDXRF) (Ferreira et al, 2012).

\subsection{TRATAMENTO DAS AMOSTRAS DE CIMENTO PARA ANÁLISE}

As amostras de cimento passaram pelo mesmo procedimento das cascas para obtenção das pastilhas para posterior análise no EDXRF.

\subsection{METODOLOGIA E ANÁLISE DAS CASCAS DE ÁRVORES E CIMENTO PELA TÉCNICA DE ESPECTROMETRIA DEFLLORESCÊNCIA DE RAIOS X POR DISPERSÃO DE ENERGIA CEDXRF]}

As curvas analíticas para determinação dos elementos químicos das cascas foram ajustadas por regressão linear e utilizado o método de Parâmetros Fundamentais para correção dos efeitos de matriz. Este método permite a obtenção da curva de sensibilidade, relacionando a intensidade fluorescente teórica calculada e a medida para cada elemento. Desta forma, é possível determinar a composição do material analisado (BONA et al, 2004). A precisão e exatidão da técnica foram verificadas usando-se o material de referência certificado de concentração similar às cascas NIST 1573a Tomato Leaves e NIST 1515 Peach Leaves que foram analisados durante todo o período das análises das cascas.

As amostras de cimento foram analisadas no modo qualitativo, fazendo uma varredura das amostras e obtendo todos os elementos constituintes do mesmo. 0 equipamento utilizado foi o EDX - 720, produzido pela Shimadzu Corporation. Os parâmetros de operação do espectrômetro foram: tubo de raios $X$ de Ródio, tensão de $15 \mathrm{KV}$; colimador de $10 \mathrm{~mm}$; detector de silício; resfriamento a nitrogênio líquido; tempo de medida de $100 \mathrm{~s}$. Os elementos determinados foram Al, Ca, Cu, Fe, K, Mg, Ni, P, S, Sr e Zn nas amostras de cascas e de cimento. 


\subsection{TRATAMENTOS ESTATÍSTICOS}

Foram calculados os fatores de enriquecimento para os elementos encontrados nas cascas da região em estudo, com o objetivo de verificar aqueles que são de inserção de atividades antropogênicas (BERLIZOV et al., 2006). Conforme reportado por Chester e outros autores (2000), quando o fator de enriquecimento for maior que dez (FE>10), a inserção é antropogênica. Para determinação dos fatores de enriquecimento foi utilizada a equação de acordo com Gresens (1967), usando Fe como agente normalizador, levando em consideração os valores de referência da crosta terrestre. Para determinação do fator de enriquecimento do elemento Fe, foi utilizado o elemento $\mathrm{Mn}$ como agente normalizador.

$\mathrm{FE}=\left(\mathrm{X}_{1} / \mathrm{Y} 1\right) /\left(\mathrm{X}_{2} / \mathrm{Y} 2\right)$, onde:

$\mathrm{X} 1$ = teor do elemento na amostra $\left(\mathrm{mg} \cdot \mathrm{kg}^{-1}\right)$;

$\mathrm{Y} 1$ = teor do Fe na amostra $\left(\mathrm{mg}_{\mathrm{kg}}{ }^{-1}\right)$;

$\mathrm{X} 2$ = teor do elemento na crosta terrestre $\left(\mathrm{mg} \cdot \mathrm{kg}^{-1}\right)$; e

$\mathrm{Y} 2$ = teor de Fe na crosta terrestre $\left(\mathrm{mg}_{\mathrm{kg}}{ }^{-1}\right)$.

Para comparação da porcentagem (doenças respiratórias/ doenças totais dos pacientes) dos povoados Machado, Estivas e Estivas II, impactados pela indústria cimenteira com a porcentagem (doenças respiratórias/ doenças totais dos pacientes) do povoado Oiteiros, localizado na mesma região, mas livre da poluição atmosférica das cimenteiras foi feito o $B o x$ Plot com a mediana das porcentagens por meio do STATISTIC 8.0 para Windows.

\subsection{MAPAS DE ATENUACÃO DAS CONCENTRAÇ̃̃ES DOS ELEMENTOS QUE POSSUEM FATOR DE ENRIQUECIMENTO MAIOR QUE DEZ [FE >10].}

A atenuação natural de um contaminante em um ecossistema é definida como um processo que ocorre no meio ambiente onde a intervenção do homem não está presente em momento algum. A massa, toxicidade, mobilidade, volume ou concentração dos contaminantes vai diminuindo naturalmente, até atingir valores naturais (WASSERMAN e QUEIROZ, 2004). 0 modelo de atenuação de concentração proposto neste trabalho baseia-se no trabalho de Ribeiro (2006) e Wasserman e Queiroz (2004).

Esse modelo consiste na distribuição espacial dos metais nos sedimentos de superfície gerando valores que descrevem a atenuação dos teores do metal a partir de um "ponto quente" ou hot spot (ponto de elevada concentração do metal) em diferentes direções, ou seja, o modelo simula a mobilidade do metal na área de estudo, neste caso, a Baia de Sepetiba, Rio de Janeiro. Ele permite avaliar, a partir de dados de concentração de um poluente, o seu comportamento em um determinado ecossistema a fim de estimar a sua mobilidade. Este modelo de atenuação foi aplicado em sistemas aquáticos utilizando dados das análises de amostras de sedimentos. Ribeiro, (2006) mostrou um panorama da situação da contaminação da Baia de Sepetiba por metais, dando uma contribuição importante ao conhecimento do comportamento e mobilidade dos metais $\mathrm{Zn}, \mathrm{Cd}, \mathrm{Pb}$, Cu e $\mathrm{Ni}$.

No estudo feito com cascas de árvores da região cimenteira foi utilizado o modelo de atenuação para avaliar a mobilidade dos elementos $\mathrm{Ca}, \mathrm{Cu}, \mathrm{S}$ e $\mathrm{Zn}$ no ar da região.

$A$ atenuação $A$ é dada em $(\mathrm{mg} / \mathrm{kg}) / \mathrm{m}$.

Nos mapas de atenuação das concentrações também foram inseridos círculos proporcionais aos problemas respiratórios da população dos povoados, a partir da porcentagem (doenças respiratórias/doenças totais dos pacientes) e georreferenciamento de suas residências por meio do programa SURFER 10.0. 


\section{RESULTADOS}

\subsection{CONTROLE ANALÍTICO DOS RESULTADOS}

O controle analítico dos resultados foi realizado por meio da análise dos materiais de referência NIST $1573^{a}$ - Tomato Leaves e Nist 1547 - Peach Leaves, provenientes do National Institute of Standards and Technology, EUA.

\subsection{FATOR DE ENRIQUECIMENTO [FE]}

Como já citado anteriormente, foram calculados os fatores de enriquecimento dos elementos encontrados nas cascas de árvores da região em estudo, com o intuito de verificar aqueles que são de inserção de atividades antropogênicas. Conforme reportado por Chester e outros autores (2000), quando o fator de enriquecimento for maior que dez (FE $>10)$, a inserção é antropogênica. Os elementos com FE> 10 foram $\mathrm{Ca}, \mathrm{Cu}, \mathrm{S}$ e Zn.

\subsection{DADOS DE SAÚDE DOS PACIENTES DOS POVOADOS MACHADO, ESTIVAS, ESTIVAS II E OITEIROS}

Foram coletados dados de saúde dos pacientes nos postos de saúde dos povoados Machado, Estivas e Estivas II totalizando 703 pacientes, sendo que as residências dos pacientes também foram georreferenciadas. Também foram coletados dados de saúde dos pacientes do povoado Oiteiros no posto de saúde do povoado, totalizando 424 pacientes.

\subsection{MAPAS DE ATENUACÃO DAS CONCENTRACÕES DOS ELEMENTOS QUE POSSUEM FE $>10$}

Figura 1 - Mapa de atenuação da concentração do Ca, com os círculos proporcionais às porcentagens de doenças respiratórias nos povoados

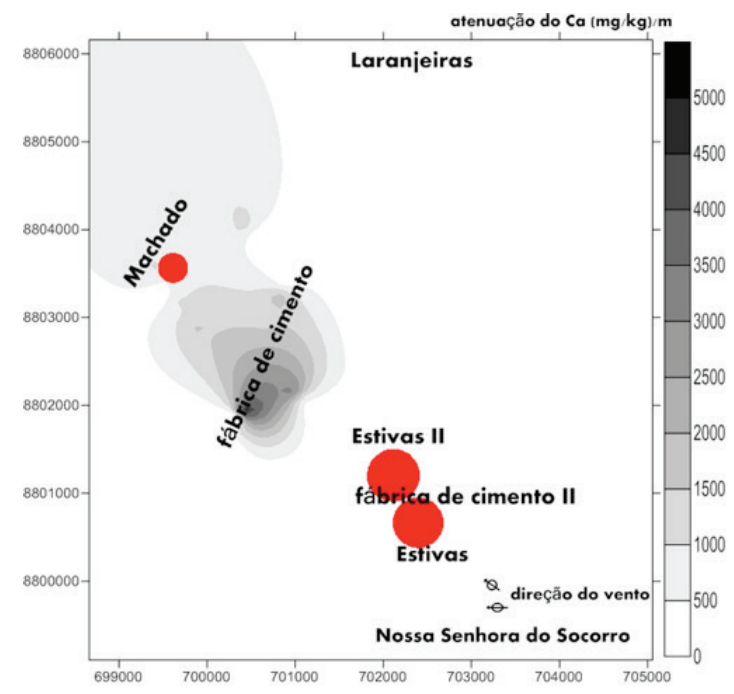

Figura 2 - Mapa de atenuação da concentração do $\mathrm{Cu}$, com os círculos proporcionais às porcentagens de doenças respiratórias nos povoados

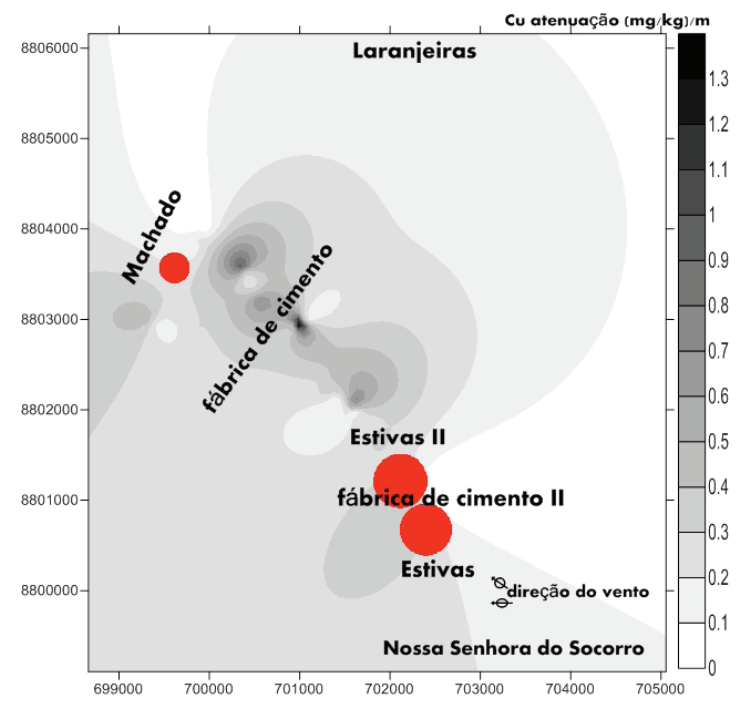


Figura 3 - Mapa de atenuação da concentração do S, com os círculos proporcionais às porcentagens de doenças respiratórias nos povoados

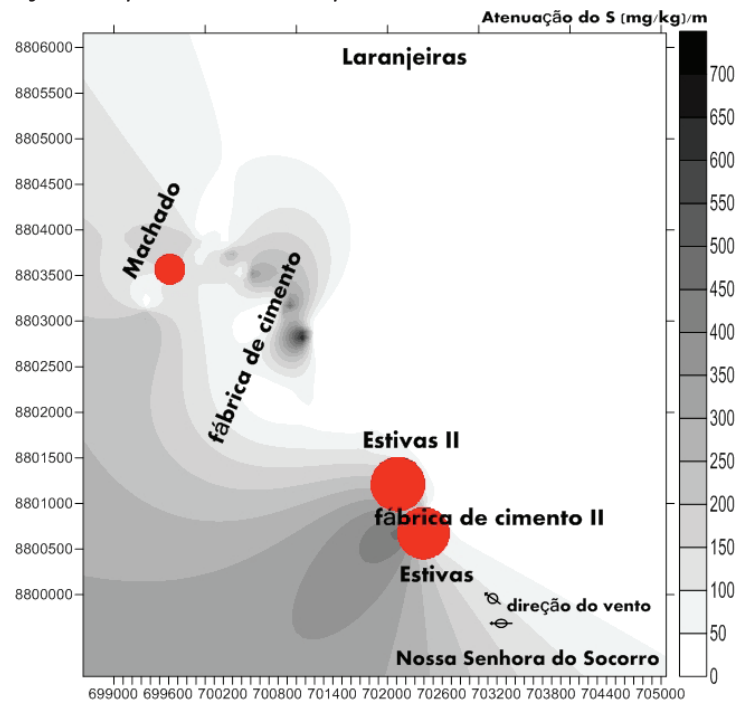

Figura 4 - Mapa de atenuação da concentração do Zn, com os círculos proporcionais às porcentagens de doenças respiratórias nos povoados

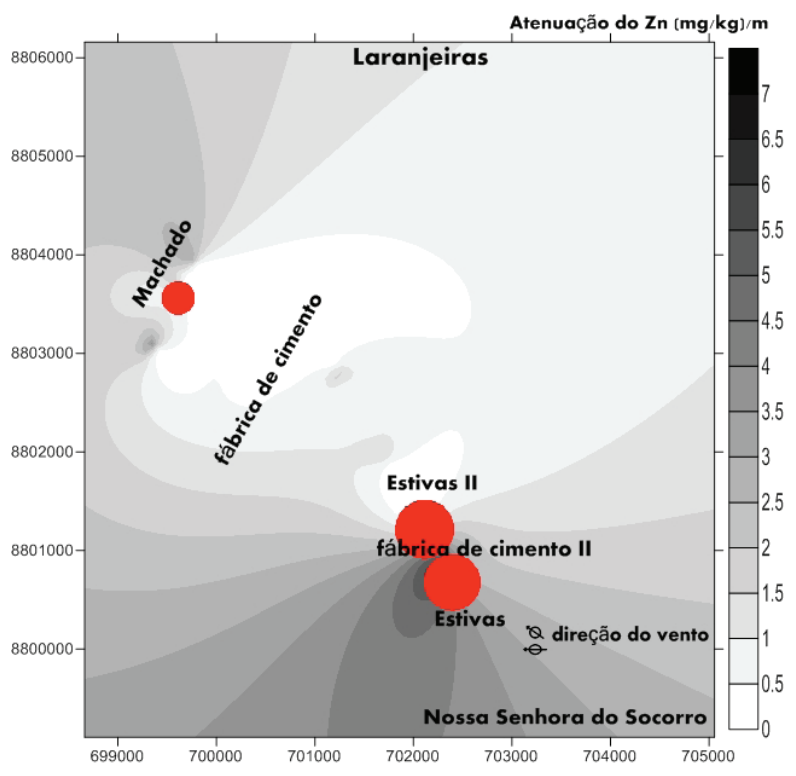

3.5 BOX PLOT COMPARANDO AS MEDIANAS DAS PORCENTAGENS CDOENCAS RESPIRATÓRIAS DO PACIENTE/ DOENCAS TOTAIS DO PACIENTEJ DOS POVOADOS MACHADO, ESTIVAS E ESTIVAS II E MEDIANAS DAS PORCENTAGENS ENCONTRADAS NO POVOADO OITEIROS

Figura 5 - Mediana da porcentagem (doença respiratória do paciente/doença total do paciente) do povoado Oiteiros comparado com a mediana encontrada nos povoados Machado, Estivas e Estivas II

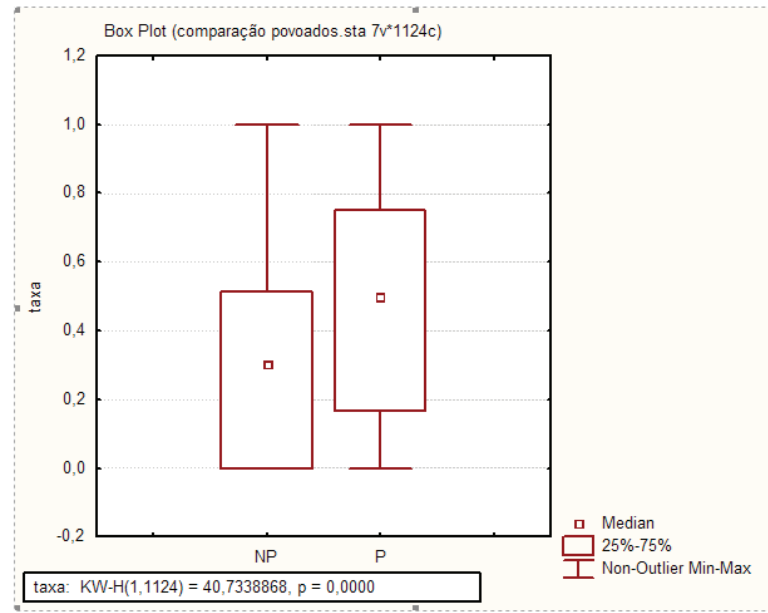




\section{DISCUSSÃO}

A exatidão e precisão das análises de casca de árvore foram verificadas por meio da análise dos materiais de referência NIST 1573a (tomato leaves) e NIST 1547 (peach leaves), fornecidos pelo National Institute of Standards of Technology, USA. Estes materiais foram analisados em repetições durante o período em que as amostras de cascas de árvores foram analisadas. Considerando os erros relativos percentuais, obteve-se que as determinações das concentrações apresentaram exatidões satisfatórias para os elementos $\mathrm{Ca}, \mathrm{K}, \mathrm{Fe}, \mathrm{P}, \mathrm{Mg}, \mathrm{Cu}$, Ni e $\mathrm{Zn}$, cujos valores encontram-se menores que $10 \%$, e exatidão relativamente satisfatórias para os elementos S, Al, e Sr cujos valores são menores ou iguais $20 \%$. As precisões das medidas avaliadas, em termos de valores de coeficiente de variação percentual, demonstraram uma repetitividade aceitável na metodologia para determinação de todos os elementos (CV\% menores que 5). Os resultados encontrados neste estudo para os materiais de referência utilizados são satisfatórios para a técnica analítica utilizada (EDXRF) (FERREIRA, MOM, 2004).

Com objetivo de identificar as regiões com inserções antrópicas, para os elementos determinados, foram mostrados os fatores de enriquecimento (FE), onde é possível verificar que $\mathrm{Ca}, \mathrm{Cu}, \mathrm{S}$ e $\mathrm{Zn}$ são oriundos de atividades industriais, uma vez que os $\mathrm{FE}>10$ para esses (CHESTER et al, 2000).

Aos resultados de concentrações de $\mathrm{Ca}$, $\mathrm{Cu}$, S e Zn encontradas em cascas de árvores foram aplicados 0 modelo de atenuação, conforme já descrito, este modelo dá as informações sobre a mobilidade do elemento, simulando o seu comportamento à medida que se afasta da sua fonte de emissão. Portanto os mapas de atenuação permitiram a identificação de áreas onde ocorre a retenção dos elementos. Os mapas de atenuação obtidos permitiram a identificação de hot spots, fornecendo informações adicionais sobre as variações de concentração na exposição da população.
0 mapa de atenuação da concentração gerado para o Ca (Figura 1) mostra um grande hot spot de atenuação na região da indústria de cimento, com grande retenção sobre o povoado Machado e Laranjeiras, não chegando ao povoado Estivas e Estivas II, podendo se inferir que esta menor área de retenção, se deva a maior granulometria do Ca que se acumula perto da fonte emissora (no caso, a área de mineração) quando comparada aos outros elementos. Os círculos indicadores de porcentagem de doenças respiratórias mostram que no Machado a proporção é menor que nos outros dois povoados, provavelmente porque sobre o povoado Machado, diferentemente de Estivas e Estivas II só há uma grande retenção do elemento Ca e não de $\mathrm{Cu}, \mathrm{S}$ e Zn. A inalação do pó de Ca pode causar irritação nas vias respiratórias, causando tosse e espirros (UNESP, 2013).

O mapa de atenuação gerado para o Cu (Figura 2) mostra que os maiores valores de atenuação deste elemento estão próximos da principal fonte de emissão deste metal (a indústria de cimento), apresentando um grande hot spot de atenuação. As informações obtidas por meio do mapa de atenuação, também, indicam que o $\mathrm{Cu}$ apresenta baixa mobilidade (maior retenção) justamente na região dos povoados Estivas e Estivas II, provavelmente devido a alguma barreira geológica, que impede sua circulação aérea.

Os círculos inseridos, feitos a partir da porcentagem (doenças respiratórias/doenças totais dos pacientes) e georreferenciamento de suas residências por meio do programa SURFER 10.0, mostra que nos povoados Estivas e Estivas II as doenças respiratórias aparecem em maior proporção que no povoado Machado. A exposição à poeira e finos de cobre pode causar irritação nos olhos, nariz e garganta, semeIhantes à gripe e associados ao sabor metálico na boca, febre e calafrios, bronco constrição e tosse. Os níveis excessivos desse metal no organismo inibem as 
enzimas que são responsáveis por proteger o organismo contra os danos provocados pelos radicais livres. A intoxicação aguda por cobre causa a erosão do epitélio gastrintestinal associado à necrose do fígado e dos rins. Além disso, o elemento cobre causa doenças ligadas à genética (MONTEIRO e MANIERI, 2009).

Em relação ao elemento $S$ sabe-se que o mesmo é um dos principais constituintes do cimento e, também, é produzido nas altas temperaturas da clinquerização. Na Figura 3 é apresentado o mapa de atenuação obtido com os dados das concentrações de $S$ encontrada em amostras de cascas de árvores. Nesta Figura 3 são observados dois hot spots de atenuação: um localizado na região da indústria, nesta região localiza-se a chaminé que funciona como uma fonte de emissão contínua de S para o meio ambiente, pois no processo de clinquerização, o cimento é aquecido a altas temperaturas, gerando entre outros, o dióxido de enxofre. Portanto, apesar de a circulação de ar ser intensa, ela não é suficiente para dissipar o $\mathrm{S}$ e ele se deposita no local e outro hot spot sobre os povoados Estivas e Estivas II, que estão localizados em uma região de baixa altitude quando comparada ao povoado Machado, no qual o $\mathrm{S}$ se deposita em menor concentração.

Como já dito anteriormente, de acordo com os círculos de porcentagem de doenças respiratórias inseridos nos mapas de atenuação, a proporção de doenças é menor em Machado e praticamente iguais em Estivas e Estivas II, corroborando o fato que nestes dois povoados há grande retenção dos elementos $\mathrm{Cu}$, $\mathrm{S}$ e $\mathrm{Zn}$, diferentemente de Machado.

Segundo a Cetesb (2013):

A principal via de exposição da população geral ao dióxido de enxofre é a inalatória. Os efeitos adversos da exposição a altos níveis de SO2 incluem dificuldade respiratória, alteração na defesa dos pulmões, agravamento de doenças respiratórias e cardiovasculares. 0 composto irrita o nariz, garganta e pulmões causando tosse, falta de ar, chiado no peito, catarro e crises de asma. Os indivíduos asmáticos ou com doenças crônicas de pulmão e coração e as crianças são mais sensíveis aos efeitos do dióxido de enxofre. Os óxidos de enxofre (SOx) podem reagir com outros compostos presentes na atmosfera, formando pequenas partículas que penetram profundamente em partes sensíveis dos pulmões, e causar ou agravar doenças respiratórias, como enfisema e bronquite, e podem agravar doença do coração preexistente, levando a internação e morte prematura. Os efeitos adversos da exposição podem ser agravados durante períodos de respiração mais rápida ou profunda, por exemplo, em exercícios físicos ou jogos, quando aumenta o volume do ar inspirado fazendo com que o SO2 penetre no trato respiratório. Estudos controlados com 26 indivíduos asmáticos praticando exercício físico indicam que algumas pessoas apresentam alterações na função pulmonar e sintomas respiratórios após curtos períodos de exposição, como 10 minutos. Outros estudos associam exposição de curto prazo ao SO2 e aumento de visitas a serviços de emergência e de internações hospitalares por doenças respiratórias, principalmente por idosos, asmáticos e crianças. A Agência Internacional de Pesquisa em Câncer (IARC) classifica o dióxido de enxofre como não classificável quanto a carcinogenicidade para seres humanos (Grupo 3). Esta categoria comumente é usada para agentes para os quais a evidência de carcinogenicidade é inadequada para o ser humano e inadequada ou limitada para animais de experimentação.

O mapa de atenuação da concentração do Zn (Figura 4) mostra maior retenção do elemento nos povoados Estivas e Estivas II, assim como o S, os quais apresentam uma maior porcentagem de doenças respiratórias. A inalação de altas concentrações de Zn podem ocasionar pneumonite tóxica e edema pulmonar (Pneumopatias ocupacionais, 2013). Para comparação de porcentagem de doenças respiratórias na população impactada pela indústria cimenteira e população de área não impactada por essas emissões aéreas, foram coletados todos os dados de saúde da população em dez anos nos postos que atendem aos povoados Machado, num total de 290 pacientes e Estivas e Estivas II, num total de 294 pacientes em Estivas e 126 em Estivas II e coletados, também, todos os dados de saúde da população em dez anos do povoado Oiteiros num total de 424 pacientes, localizado como Estivas e Estivas II no município de Nossa Senhora do Socorro, mas livre das emissões aéreas da fábrica, sentindo apenas o tremor das explosões das dinamites na mina. 
Para cada paciente foi feita a equação ((total de doenças respiratórias)/(total de doenças)) e encontrada a porcentagem de doenças respiratórias para cada paciente. Por meio do ESTATISTIC 8.0 foi feito o Box Plot das medianas das porcentagens de doenças respiratórias encontradas na região impactada e não

\section{CONCLUSÃO}

A partir dos resultados, pode-se concluir que a utilização de cascas de árvores como bioindicador é uma estratégia adequada em estudos sobre impactos ambientais. $\mathrm{O}$ uso de cascas de árvores no presente estudo foi abrangente, preciso, de baixo custo e de fácil implementação. É importante que as estratégias desenvolvidas para estimar o nível de contaminação não dependam de métodos instrumentais sofisticados, envolvendo enormes recursos para sua implantação, e, sobretudo, longos prazo de manutenção.

Neste contexto a abordagem empregada neste estudo pode ser útil para a análise ambiental em áreas com emissões aéreas e sem redes convencionais de monitoramento. Os mapas de atenuação da distribuição das concentrações dos elementos ajudaram a localizar hot spots de poluição e a estimar a mobilidade destes elementos no ar, mostrando a concentração de impactada. Ele mostra que $50 \%$ dos pacientes que procuram os postos de saúde nos povoados Machado, Estivas e Estivas II são devidos às doenças respiratórias, diferentemente do povoado Oiteiros onde somente $30 \%$ dos pacientes que procuram o posto de saúde são devidos a essas doenças.

poluentes as quais a população está exposta e apresentando a vantagem de ser também uma metodologia de baixo custo e de ser uma ferramenta auxiliar para interpretar o comportamento dos elementos químicos. A partir dos resultados pode-se concluir que a indústria de cimento é impactante para o ar em toda área vizinha ao complexo cimenteiro.

É necessário, devido aos resultados aqui demonstrados que sejam efetuados estudos epidemiológicos, importantes para proteção da saúde e estabelecimento de medidas mitigadoras para esta população exposta às concentrações mais elevadas destes poluentes.

Os resultados obtidos na aplicação da técnica do EDXRF foram eficientes na caracterização do material particulado proveniente das fontes poluidoras.

\section{REFERÊNCIAS}

BERLIZOV A N, BLUM O B, FILBY R H, MALYUK I A, TRYSHYN V V. Testing applicability of black poplar (Populus nigra L.) bark to heavy metal air pollution monitoring in urban and industrial regions, Sci Environ 372 (2007) 693-706, 2006.

BONA IAT, SARKIS LES, SALVADOR VLR, SOARES ALR, KLMT SC. Análise arqueométrica de cerâmica Tupiguarani da região central do Estado do Rio Grande do
Sul, Brasil, usando fluorescência de raios $X$ por dispersão de energia (EDXRF), Quím Nova, v. 30, n. 4, São Paulo, 2004.

CETESB, 2013 - Companhia de Tecnologia de Saneamento Ambiental do Estado de São Paulo. Disponível em: <http://www.cetesb.sp.gov.br/userfiles/file/laboratorios/fit/dioxido_de_enxofre.pdf>. Acesso em: $10 / 03 / 2013$ 
CHESTER R, NIMMO M, FONES GR, KEYSE S, ZHANG $Z$. Trace metal chemistry of particulate aerosols from the UK mainland coastal rim of the NE Irish sea, Atmos Environ 34, 949-958, 2000.

FERREIRA AB, SANTOS JO, SOUZA SO, SANTOS Jr WN, ALVES, JPH. Use of passive biomonitoring to evaluate the environmental impact of emissions from cement industries in Sergipe State, northeast Brazil, MICROCHEMICAL JOURNAL, 103, 15-20, 2012.

FERREIRA, MOM. Determinação de componentes inorgânicos em plantas medicinais, comercializadas em formas de pó (cápsulas) e "in natura", utilizando a técnica de fluorescência de raios $x$ por dispersão de comprimento de onda (WDXRF) e por dispersão de energia (EDXRF). Definição de perfis inorgânicos quantitativos. [Tese], São Paulo: Instituto de Pesquisas Energéticas e Nucleares, Universidade de São Paulo, 2004.

GRESENS RL, Composition-volume relationships of metasomatism, Chem Geol Amsterdam, v. 2, 1967, 47-55.

IBGE (2011) - Instituto Brasileiro de Geografia e Estatística. Disponível em: <http://www.ibge.gov.br/ home/mapa_site/mapa_site.php\#populacao>. Acesso em: $10 / 02 / 2013$

MONTEIRO, LPC; MAINIER, FB. Experimentos de lixiviação de metais em corpos-de-prova de cimento branco relacionados com a possível contaminação do cimento produzido a partir da queima de resíduos industriais. V. 11, n. 1, julho, 2009, p. 24-31.
Pneumopatias ocupacionais (2013). Disponível em: <http://www.sociedadeclementeferreira.org.br/images/Caracteristicas_gravidade_pneumopatias_ocupacionais.pdf>. Acesso em: 15/03/2013

RIBEIRO, AP. Procedimento de fracionamento comparado a Modelo de Atenuação para a avaliação de mobilidade de metais pesados em sedimentos da Baía de Sepetiba, Rio de Janeiro. [Tese]. São Paulo: Instituto de Pesquisas Energéticas e Nucleares, Universidade de São Paulo, 2006.

SILVA BB, ALVES JJA, CAVALCANTI EP, DANTAS RT. Potencial eólico na direção predominante do vento no Nordeste brasileiro. Revista brasileira de engenharia agrícola e ambiental, v. 6, n. 3, Campina Grande, 2002, 431-439.

SCHELLE E, STATON I, CLARKSON P, BELLIS DJ, MC LEOD CW. Rapid multielement analysis of tree bark by EDXRF, J Environ Anal Chem, 2001, 82:785-793.

Unesp (2013) - Universidade do Estado de São Paulo. Disponível em: <www.qca.ibilce.unesp.br/prevencao/produtos/cloreto_calcio.html>. Acesso em: $15 / 03 / 2013$

WASSERMAN JC, QUEIROZ EL. The attenuation of concentrations model: a new method for assessing mercury mobility in sediments, Quím. Nova, 2004, 27:17-21. 7. 
1 Instituto Tecnológico e de Pesquisas do Estado de Sergipe (ITPS), Rua Campo do Brito, 371, Aracaju, SE, 6, Brasil. E-mail: angelicaferreira17@ yahoo.com.br

2 Instituto Federal de Ciência e Tecnologia de Sergipe, Av. Eng ${ }^{\circ}$. Gentil Tavares da Motta, 1166, Aracaju, 8 SE, Brasil. E-mail: osmansantos@ig.com.br

3 Instituto Tecnológico e de Pesquisas do Estado de Sergipe (ITPS), Rua Campo do Brito, 371, Aracaju, SE, 6, Brasil. E-mail: sidnei.ufs@hotmail.com

4 Instituto Tecnológico e de Pesquisas do Estado de Sergipe (ITPS), Rua Campo do Brito, 371, Aracaju, SE, 6, Brasil. E-mail: wil_jun@hotmail.com

5 Laboratório de Química Analítica Ambiental, Departamento de Química da Universidade Federal de Sergipe, Av. Marechal Rondon, 81, Aracaju, SE, Brazil. E-mail: patrocínio@itps.se.gov.br 